\title{
Aplicaciones prácticas de la espectroscopía de absorción infrarroja en el estudio de los crudos, del clínker y del cemento portland anhidro
}

(Continuación)

TOMAS VAZQUEZ, Dr. en Ciencias Químicas

I.E.T.e.c.

\subsubsection{Vibraciones $\mathrm{OH}$ y $\mathrm{H}-\mathrm{O}-\mathrm{H}$}

Para el estudio de este tipo de vibraciones, a continuación se resume el trabajo de C. ROCCHICCIOLI (10) "La spectrographia d'absorption infrarouge, moyen d'étude de l'eau dans les substances minérales".

Las diferentes clases de agua en sustancias minerales pueden clasificarse en:

a) Agua de absorción.

b) Agua de imbibición, zeolítica.

c) Agua de cristalización y agua de constitución.

Como regla general se puede decir: en una sustancia hidratada las moléculas de agua están tanto más libres cuanto más elevadas están las frecuencias de vibración de valencia $\mathrm{OH}$ y más bajas las de deformación $\mathrm{H}-\mathrm{O}-\mathrm{H}$, en el espectro IR correspondiente.

Las vibraciones fundamentales del agua son tres: dos de ellas de valencia $\left(\begin{array}{lll}\nu_{1} & \text { y } & \nu_{3}\end{array}\right)$ y una de deformación $\left(\nu_{2}\right)$ (fig. 1).

Las bandas de absorción, aparecen a diferentes frecuencias dependiendo de numerosos factores (agua vapor, líquida, sólida; fuerza de unión del agua con el resto de la molécula, etc.). A título orientativo se dan tres frecuencias de vibración correspondientes al agua líquida :

- (vibración de valencia simétrica) $3.450 \mathrm{~cm}^{-1}$;

- (vibración de deformación) $\quad 1.640 \mathrm{~cm}^{-1}$;

- (vibración de valencia asimétrica) $3.580 \mathrm{~cm}^{-1}$. 

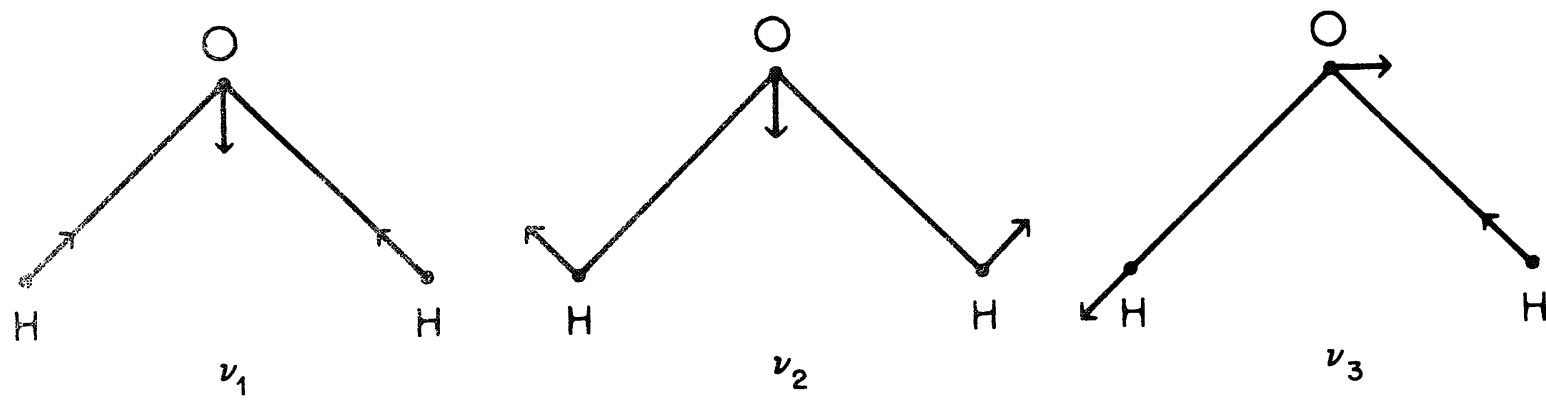

Fig. 1.-Vibraciones fundamentales del agua.

\section{a) Agua de absorción}

Cono este tipo de agua es muy libre, su espectro IR da unas bandas de absonción de valencia simétrica y asimétrica $\left(\nu_{1}\right.$ y $\left.\nu_{3}\right)$ en números de onda más altos que los correspondientes a agua líquida, y una banda debida a vibración de deformación a frecuencia más beja.

\section{b) Agua de imbibición, agua zeolitica}

Corresponde a un tipo de agua que el mineral puede pender y recuperar con cierta facilidad. Sus bandas de vibración se comportan, generalmente, de forma similar a las del agua de absorción: aparecen a números de onda más elevados para $\nu_{2}$ y más bajos para $\nu_{1}$ y $y_{s}$.

\section{c) Agua de cristalización y agua de constitución}

Aunque no es posible trazar un límite claro entre agua de cristalización y de constitución, la espectroscopía IR contribuye decisivamente en la distinción de uno y otro tipo. Se puede hacer a través de:

- Localización de las bandas características. El agua de constitución está mucho más ligado que el de cristalización y, por consiguiente, las bandas de valencia simétrica y asimétrica aparecen en el espectro IR a números de onda más bajos que el agua de cristalización.

- La influencia ejencida por uno y otro tipo de agua sobre el resto de la molécula. Si se deshidrata un compuesto que contiene agua de constitución queda afectado de tal manera que produce un espectro IR con modificaciones de importancia respecto al de la sustancia no deshidratada.

- El comportamiento de un compuesto con agua de cristalización sería distinto: deshidratado daría un espectro IR con alteraciones relativamente pequeñas.

\subsubsection{Conclusiones prácticas}

Como resumen del capítulo estudiado se podría decir:

Referente a la caliza:

- La espectroscopía IR puede aplicarse de una forma rápida y eficaz al estudio cuali y cuantitativo del $\mathrm{CaCO}_{3}$, en cualquiera de sus formas oristalinas. 
- Una sustancia es tanto más pura cuanto su espectro IR da menor número de bandas. $\mathrm{Y}$, en general, tanto mejor cristalizada cuanto más definidas aparezcan sus bandas de absorción.

Referente a la arcilla:

- Como la espectrografía IR da la "huella dactilar" de una sustancia, esté o no cristalizada, mediante sencilla comparación de espectros se conocerá el tipo de arcilla que se está utilizando, así como el grado de su pureza. Para ello es necesario la existencia previa del espectro IR de la muestra en estudio. En el caso de no disponer de él, el espectro infrarrojo da una orientación muy eficaz respecto a los distintos tipos de grupos estructurales y, en consecuencia, de la composición cualitativa de la sustancia en estudio.

- La espectroscopía IR informa sobre la cantidad y modo de estar ligada el agua en la estructura de la arcilla.

\section{ESTUDIO DEL CLINKER PORTLAND POR IR}

En el presente capítulo nos referimos a las fases principales del clínker: alita, belita, fase ferrítica y fase alumínica.

\subsection{Alita}

La fase más importante del clínker portland es la conocida como "alita" y que esencialmente es $\mathrm{C}_{3} \mathrm{~S}$.

Los modos normales de vibración de la molécula tetraédrica $\mathrm{SiO}_{4}$ han sido estudiados en 2.2.1. Aquí nos limitaremos a exponer las frecuencias de vibración y las correspondientes asignaciones, del grupo $\mathrm{SiO}_{4}$ en el caso del $\mathrm{C}_{3} \mathrm{~S}$.

\begin{tabular}{cccc}
\hline FRECUENCI $\left(\mathrm{cm}^{-1}\right)$ & ASIGNACION & FRECUENCIA $\left(\mathrm{cm}^{-1}\right)$ & ASIGNACION \\
\hline $950 \mathrm{~F}$ & & $525 \mathrm{f}$ & \\
$892 \mathrm{~F}$ & $\nu_{3}-\mathrm{SiO}_{4}$ & $450 \mathrm{~m}$ & $\nu_{4}-\mathrm{SiO}_{4}$ \\
$810 \mathrm{~h}$ & $\nu_{1}-\mathrm{SiO}_{4}$ & $360 \mathrm{~h}$ & $\nu_{2}-\mathrm{SiO}_{4}$ \\
& & $340 \mathrm{f}$ & \\
\hline
\end{tabular}

( $F=$ muy fuerte $; f=$ fuerte $; m=$ media $; \mathrm{h}=$ "hombro").

La alita se forma en el proceso de clinkerización y admite en disolución sólida, como impurezas o como sustituciones isomórficas, numerosos elementos y compuestos. Las alitas que se encuentran formando parte del clínker son, generalmente, de formas monoclínicas y trigonales del $\mathrm{C}_{3} \mathrm{~S}$ [GUINIER y REGOURD (12), YAMAGUCHI y UCHIKAWA (13)]. Hay autores [BENSTED y PRAKASH VARMA (14)] que indican que son más frecuentes las formas del monoclínico y triclínico.

Se puede decir estrictamente que existen infinidad de tipos de alita, según la naturaleza y cantidad de iones extraños que el $\mathrm{C}_{3} \mathrm{~S}$ admita. En todo caso las variaciones que se reflejan en los espectros IR son de poca importancia, aunque significativas; en general cuanto 


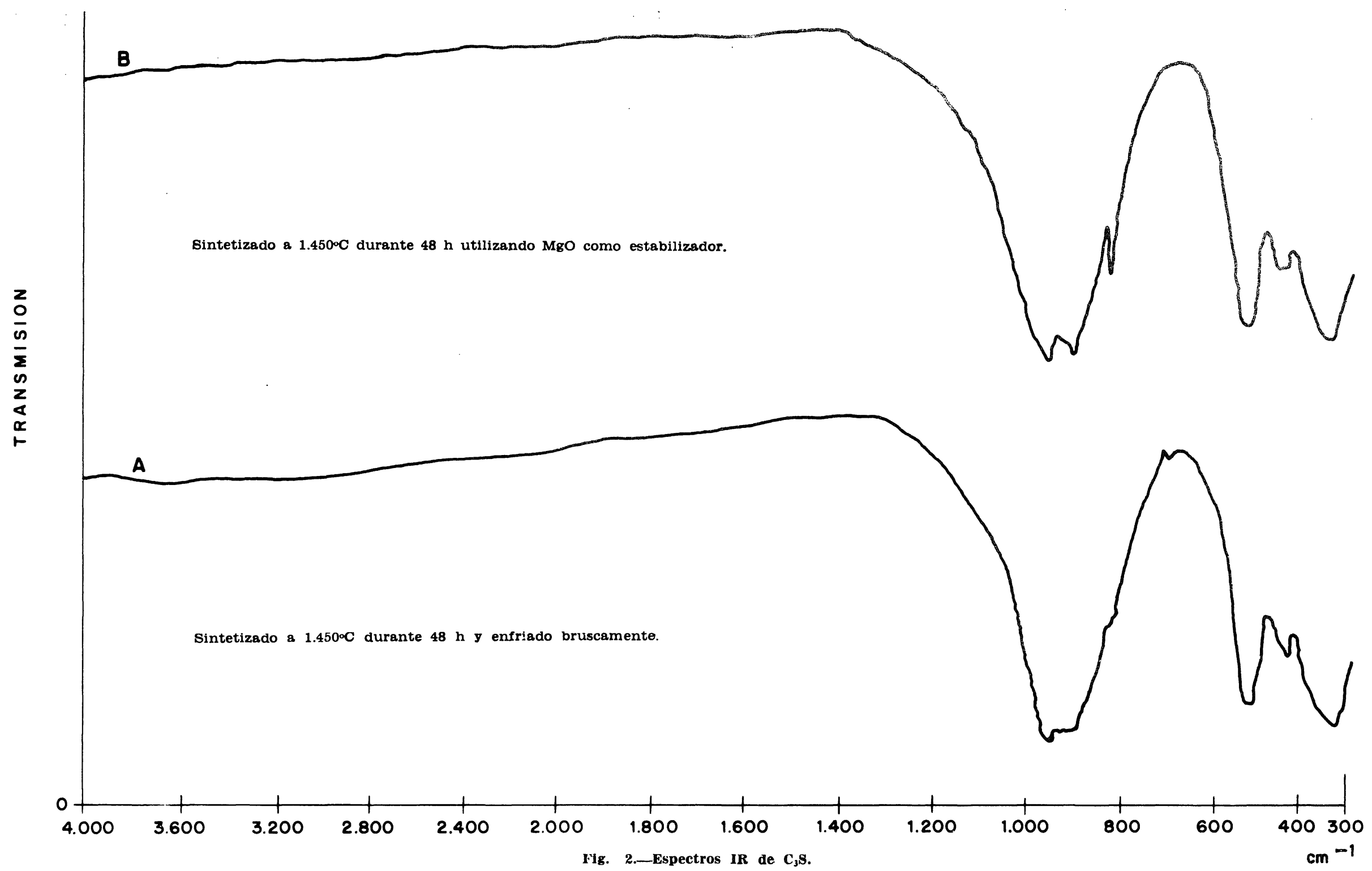


más puro es el $\mathrm{C}_{3} \mathrm{~S}$ las bandas $\mathrm{IR}$ aparecen más agudas y definidas. Como ya se ha dicho, en este mismo sentido influye la mayor o menor cristalinidad de la muestra, el "templado" o "recocido" que haya sufrido. La banda de absorción que aparece en el espectro en $810 \mathrm{~cm}^{-1}$ es aguda si se trata de una muestra con cristalinidad alta y aparece en forma de "hombro" con las de baja cristalinidad (fig. 2).

\subsection{Belita}

El $\mathrm{C}_{2} \mathrm{~S}$ puede presentarse en las formas conocidas como $\alpha, \alpha^{\prime}, \beta$ y $\gamma$. Para el estudio del clínker portland la más importante es la $\beta$, al ser la forma que se asimila a la belita. Cristaliza en el sistema monoclínico. Tiene propiedades hidráulicas y, aun cuando puro, no sería estable a temperatura ambiente, se estabiliza con facilidad mediante impurezas e incluso por el tamaño del cristal. La inestabilidad del $\beta-C_{2} \mathrm{~S}$ llevaría a su transformación a la forma $\gamma$ (que es la modificación cristalina estable a temperatura ambiente), no hidráulica, con autopulverización y fuerte expansión.

Al igual que la alita, la fase belítica admite iones extraños en su red. Los correspondientes espectros, según BENSTED y PRAKASH VARMA (14), sufrirían muy pocas variaciones.

En nuestra opinión, consideramos que el espectro IR del $\beta-C_{2} \mathrm{~S}$ no está aún perfectamente definido, quizás precisamente a causa de los diferentes métodos empleados para su síntesis.

En un anterior trabajo (15) dábamos como valores característicos del espectro IR del $\beta-C_{2} S$ los siguientes:

\begin{tabular}{cccc}
\hline FRECUENCIA $\left(\mathbf{c m}^{-1}\right)$ & ASIGNACION & FRECUENCIA $\left(\mathbf{c m}^{-1}\right)$ & ASIGNACION \\
\hline $1.080 \mathrm{D}$ & $2 \times \nu_{4}$ & $878 \mathrm{~F}$ & $\nu_{3}$ \\
$992 \mathrm{f}$ & $\nu_{3}$ & $848 \mathrm{f}$ & $\nu_{1}$ \\
$970 \mathrm{~h}$ & & $540 \mathrm{~h}$ & $\nu_{4}$ \\
$935 \mathrm{~F}$ & $\nu_{3}$ & $520 \mathrm{~F}$ & $\nu_{4}$ \\
$914 \mathrm{~F}$ & $\nu_{3}$ & $508 \mathrm{~h}$ & $\nu_{4}$ \\
& & $370 \mathrm{~h}$ & $\nu_{2}$ \\
& & $300 \mathrm{~F}$ & $\nu_{2}$ \\
\hline
\end{tabular}

Los valores dados tienen alguna dispersiỏn respecto a los que BENSTED y PRAKASH VARMA obtienen (14). Probablemente el motivo principal de esas diferencias son los distintos métodos de síntesis de la sustancia seguidos, además de la naturaleza del espectro (con bandas anchas, poco definidas) que hace difícil la precisión de valores.

En todo caso incluimos aquí las frecuencias que dan aquellos autores al $\beta-\mathrm{C}_{22} \mathrm{~S}$ :

\begin{tabular}{rccc}
\hline FRECUENCIA $\left(\mathbf{c m}^{-1}\right)$ & ASIGNACION & FRECUENCIA (cm-1) & ASIGNACION \\
\hline $1.170 \mathrm{D}$ & $2 \times \nu_{4}-\mathrm{SiO}_{4}$ & $540 \mathrm{~h}$ \\
$1.000 \mathrm{f}$ \\
$920 \mathrm{~h}$ \\
$890 \mathrm{~F}$ \\
$870 \mathrm{~h}$ \\
$845 \mathrm{~F}$
\end{tabular}


En la figura 3 se dan los espectros IR de 4 muestras de $\beta-C_{2} S$, en los cuales se aprecian diferencias que de algún modo pueden justificar los valores no coincidentes obtenidos. Todas las muestras han sido comprobadas previamente por difracción de rayos X y ATD.

Asimismo juzgamos interesante el incluir aquí el espectro IR (fig. 4) que corresponde a una muestra (presuntamente $\beta-C_{2} S$ ) sintetizada a partir de compuestos puros $\left(\mathrm{CaCO}_{3}\right.$ y $\mathrm{SiO}_{2}$ gel) a una temperatura de $1.000^{\circ} \mathrm{C}$ durante 4 días. Rayos $\mathrm{X}$ da un difractograma característico de $\beta-C_{2} \mathrm{~S}$ y algo de $\mathrm{CaO}$ y por análisis térmico se interpreta que se trata de una mezcla de $\mathrm{C}_{2} \mathrm{~S}$ puro, $\mathrm{C}_{2} \mathrm{~S}$ y $\mathrm{CaO}$ (en disolución sólida) y algo de $\mathrm{Ca}(\mathrm{OH})_{2}$ (de meteorización de $\mathrm{CaO}$ ).

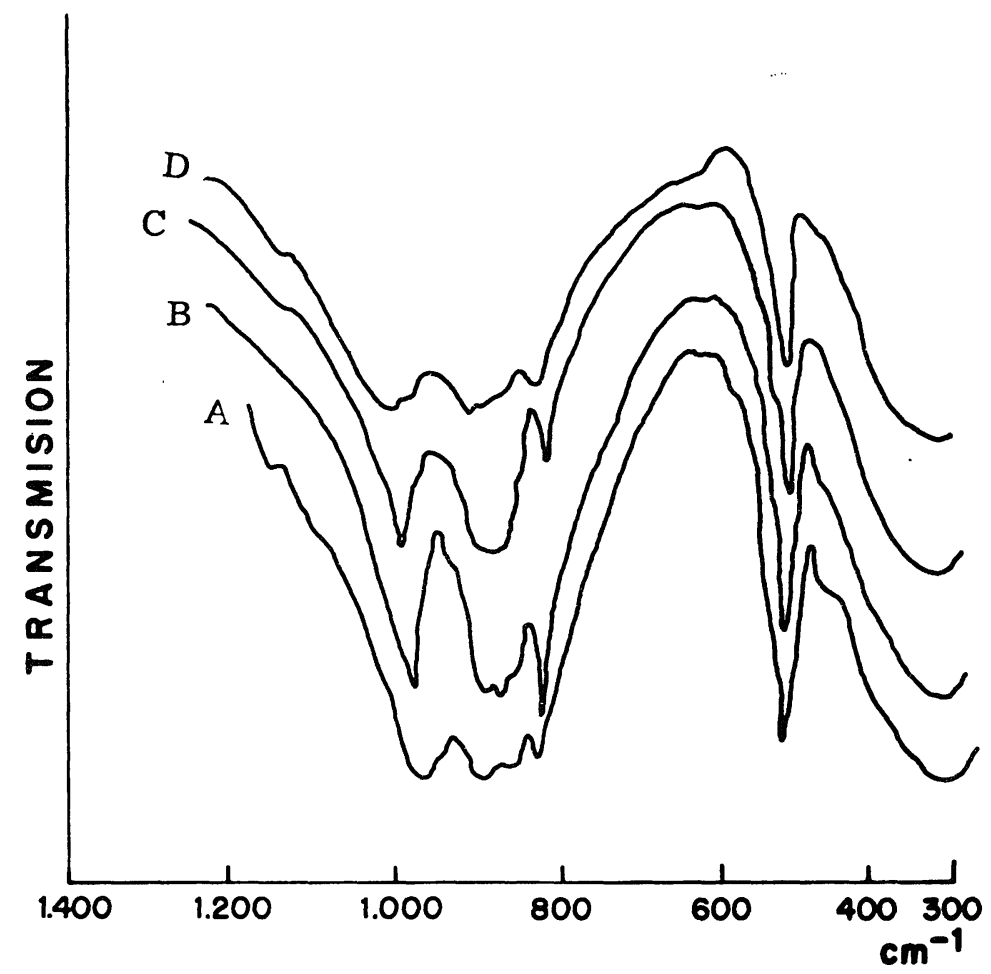

Fig. 3.-Espectros IR de compuestos que presuntamente son $\beta-C_{2} \mathbf{S}$.
$\mathbf{A}=$ Estabilizado con $\mathrm{K}^{+}(i \mathrm{con}$ estructura intermedia de $\beta-C_{2} \mathrm{~S}$ y $\quad \mathrm{KC}_{23} \dot{\mathrm{S}}_{12}$ ?)

$\mathbf{B}=$ Calentado durante 4 días $\mathrm{a}$ $1.000^{\circ} \mathrm{C}$, sucesivamente.

$\mathrm{C}=$ Con $\mathrm{B}_{2} \mathrm{O}_{3}$ como estabilizador molido y calentado a $1.500^{\circ} \mathrm{C}$ cuatro veces, sucesivamente. Posiblemente haya $\mathrm{CaO}$ disuelta en el $\beta-C_{2} s$.

$\mathrm{D}=$ Estabilizado con $\mathrm{B}_{2} \mathrm{O}_{3}$. Calentado durante 24 h a

Por el estudio del espectrograma de IR (pág. 51) damos la interpretación siguiente:

Se trata de una mezcla de sustancias, entre las que aparece $\mathrm{Ca}(\mathrm{OH})_{2}$ (banda aguda en $3.640 \mathrm{~cm}^{-1}$ ), silicatos del tipo $\mathrm{Si}_{2} \mathrm{O}_{7}$-sorosilicatos- (bandas en 646 y $1.100 \mathrm{~cm}^{-1}$ ) e impurezas de silicatos del tipo seudowollastonita - ciclos $\mathrm{Si}_{3} \mathrm{O}_{9}-\left(720 \mathrm{~cm}^{-1}\right)$. Las bandas restantes caracterizarían al $\beta-C_{2} S$, y coinciden sensiblemente con las indicadas por BENSTED y PRAKASH VARMA.

\subsection{Fase ferrítica}

Como simplificación para su estudio, la fase ferrítica se ha considerado tradicionalmente como $\mathrm{C}_{4} \mathrm{AF}$. Realmente se trata de una disolución sólida cuya fórmula se representaría como $C_{2}\left(A_{1-x}, F_{x}\right)$ en la cual $x$ puede variar entre 1 y 0,33 (16). Esta fase admite en su estructura iones extraños, al igual que el resto de las que componen el clínker portland. La formación estructural de la fase ferrítica es fácilmente imaginable tomando como re- 


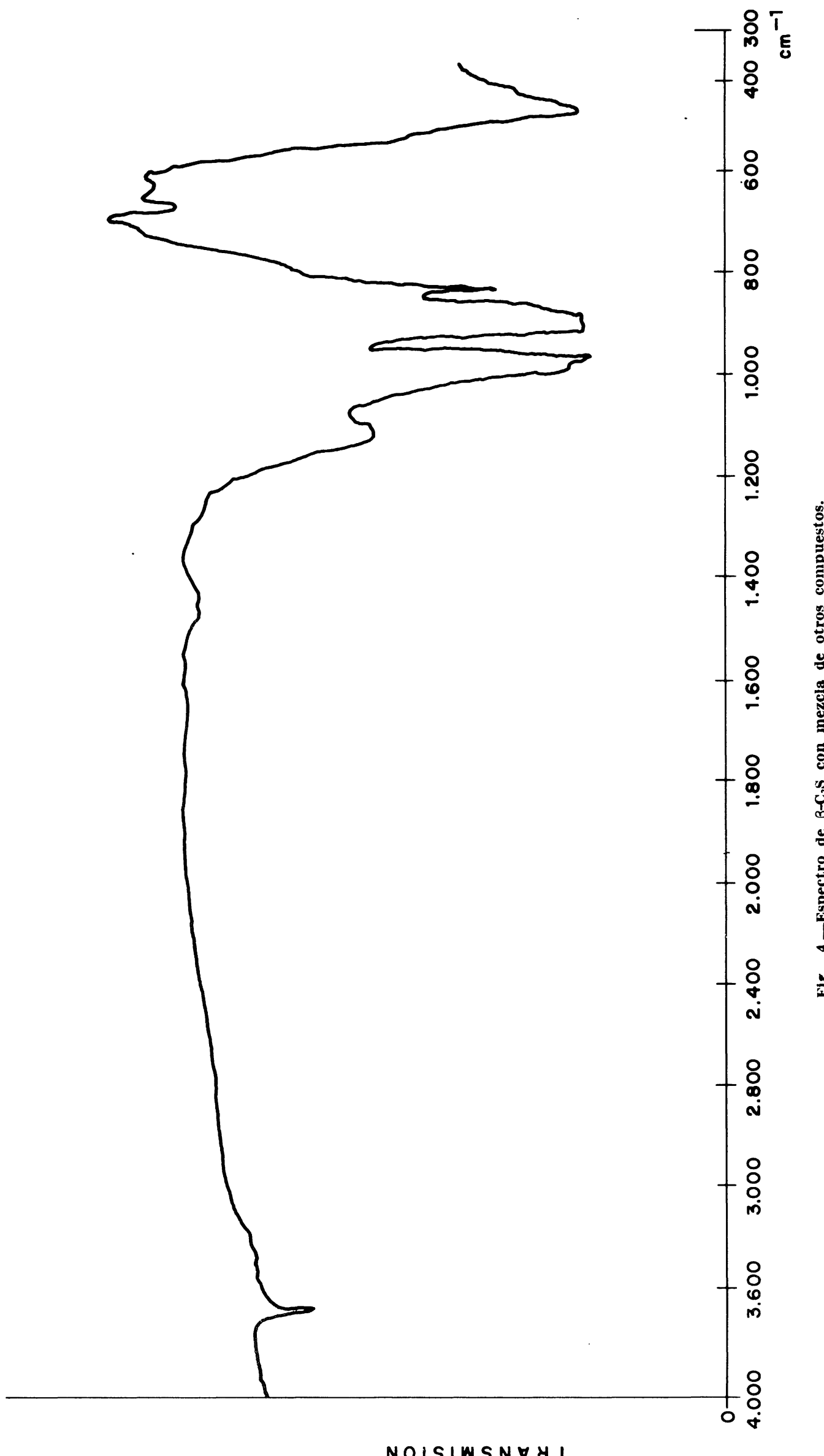

51

(C) Consejo Superior de Investigaciones Científicas

http://materconstrucc.revistas.csic.es

Licencia Creative Commons 3.0 España (by-nc) 
ferencia el $\mathrm{C}_{2} \mathrm{~F}$ (fig. 5). En este compuesto el hierro ocupa lugares tetra y octaédricos. En ambas posiciones puede ser sustituido por el aluminio. El reemplazamiento inicial se realiza sobre posiciones tetraédricas $\mathrm{y}$, posteriormente en compuestos más ricos en $\mathrm{Al}$, también en posiciones octaédricas.
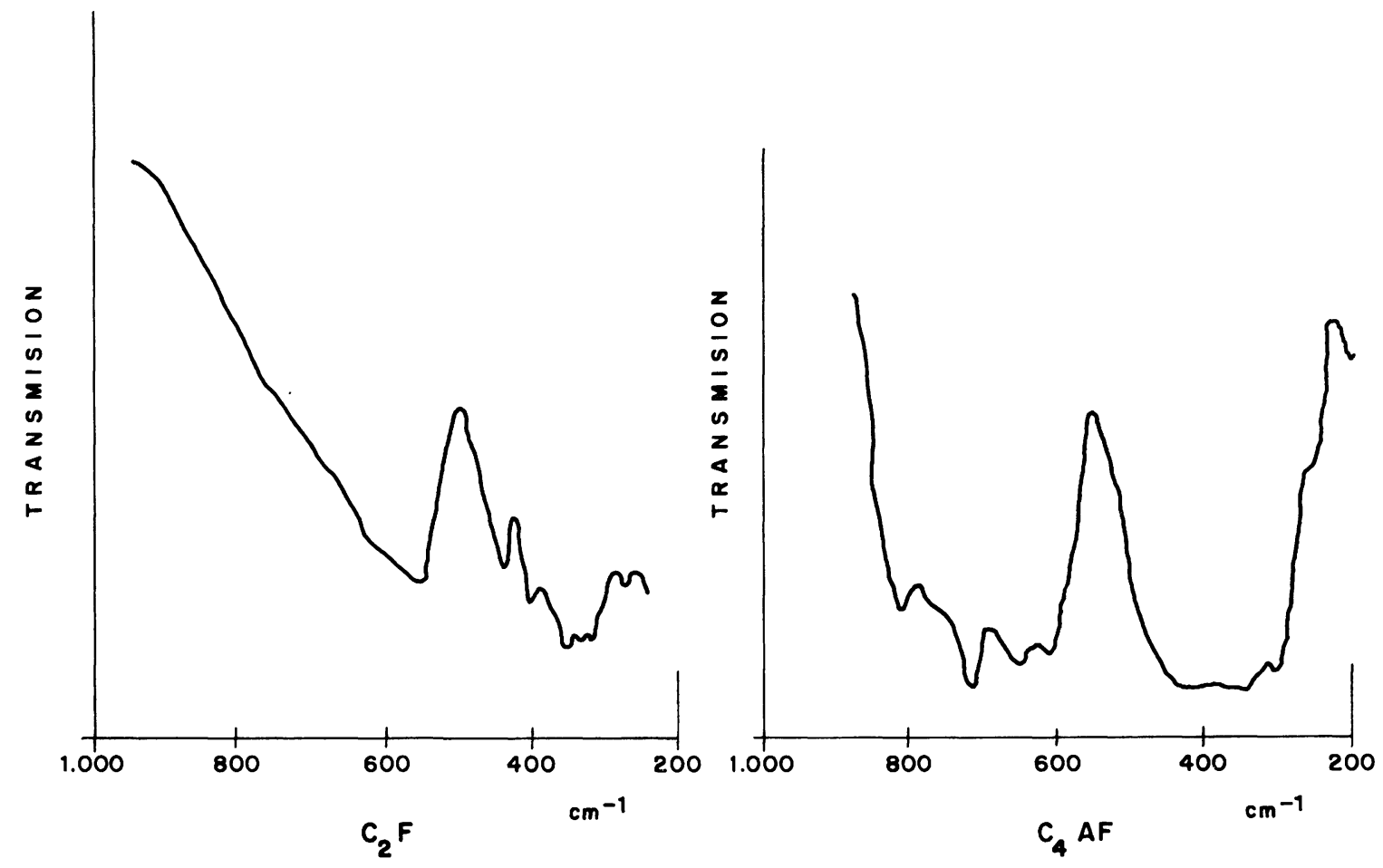

Fig. 5

Los valores característicos, en $\mathrm{cm}^{-1}$, del $\mathrm{C}_{4} \mathrm{AF}$ son los siguientes:

\begin{tabular}{cccc}
\hline AlO $_{4}$ & FeO $_{4}$ & AlO $_{6}$ & FeO $_{6}$ \\
\hline 810 & 650 & 570 & 410 \\
760 & & 520 & $328 ?$ \\
718 & 610 & 440 & $310 ?$ \\
\hline
\end{tabular}

Los modos normales de vibración de las moléculas octaédricas y tetraédricas ya se han representado en 2.2.1. $y^{\dagger}$ 2.2.2. En el cuadro 1 se dan, de acuendo con TARTE $(16,17)$ las vibraciones características de los tetraedros $\mathrm{AlO}_{4}$ de los octaedros $\mathrm{AlO}_{6}$, cuando se encuentran aislados y "condensados" y de los tetra y octaedros $\mathrm{FeO}_{4}$ y $\mathrm{FeO}_{6}$.

CUADRO 1

\begin{tabular}{|c|c|c|c|c|}
\hline \multirow{3}{*}{ 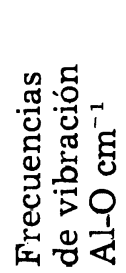 } & $\begin{array}{c}\text { A10 } \\
\text { Tetraédrico }\end{array}$ & $\begin{array}{c}\mathrm{AlO}_{6} \\
\text { Octaédrico }\end{array}$ & $\begin{array}{c}\text { FeO, } \\
\text { Tetraédrico }\end{array}$ & $\begin{array}{c}\text { FeO }_{6} \\
\text { octaédrico }\end{array}$ \\
\hline & $\begin{array}{c}\text { Condensado: } \\
900-700\end{array}$ & $\begin{array}{c}\text { Condensado: } \\
680-500\end{array}$ & $\begin{array}{c}\text { Condensado: } \\
\left({ }^{*}\right)\end{array}$ & $\begin{array}{c}\text { Condensado } \\
\left({ }^{*}\right)\end{array}$ \\
\hline & $\begin{array}{c}\text { Aislado: } \\
800-650\end{array}$ & $\begin{array}{l}\text { Aislado: } \\
530-400\end{array}$ & $\begin{array}{l}\text { Aislado: } \\
\text { 650-600 }\end{array}$ & $\begin{array}{l}\text { Aislado: } \\
\text { 500-350 }\end{array}$ \\
\hline
\end{tabular}

(*) Los grupos $\mathrm{FeO}_{4}$ y $\mathrm{FeO}_{6}$, cuando están “condensados”, dan bandas que pueden estar desplazadas respecto a las de los grupos "aislados" más de $150 \mathrm{~cm}^{-1}$ hacia mayor frecuencia, al igual que los grupos AlO,-AlO, 


\subsection{Fase alumínica}

En la simplificación del estudio de los aluminatos del clínker portland, así como para el cálculo potencial de Bogue, se considera que están como $\mathrm{C}_{3} \mathrm{~A}$ (además, naturalmente de los integrados en la fase ferrítica). La estructura del $\mathrm{C}_{3} \mathrm{~A}$ no ha sido confirmada plenamente hasta ahora, aunque la opinión más generalizada es la de que se trata de una estructura cúbica, con coordinación 4 para el Al.

En base a esta coordinación damos en el cuadro 2 las frecuencias de vibración y las correspondientes asignaciones de las bandas de absorción del espectro del $\mathbf{C}_{3} \mathbf{A}$ (fig. 6).

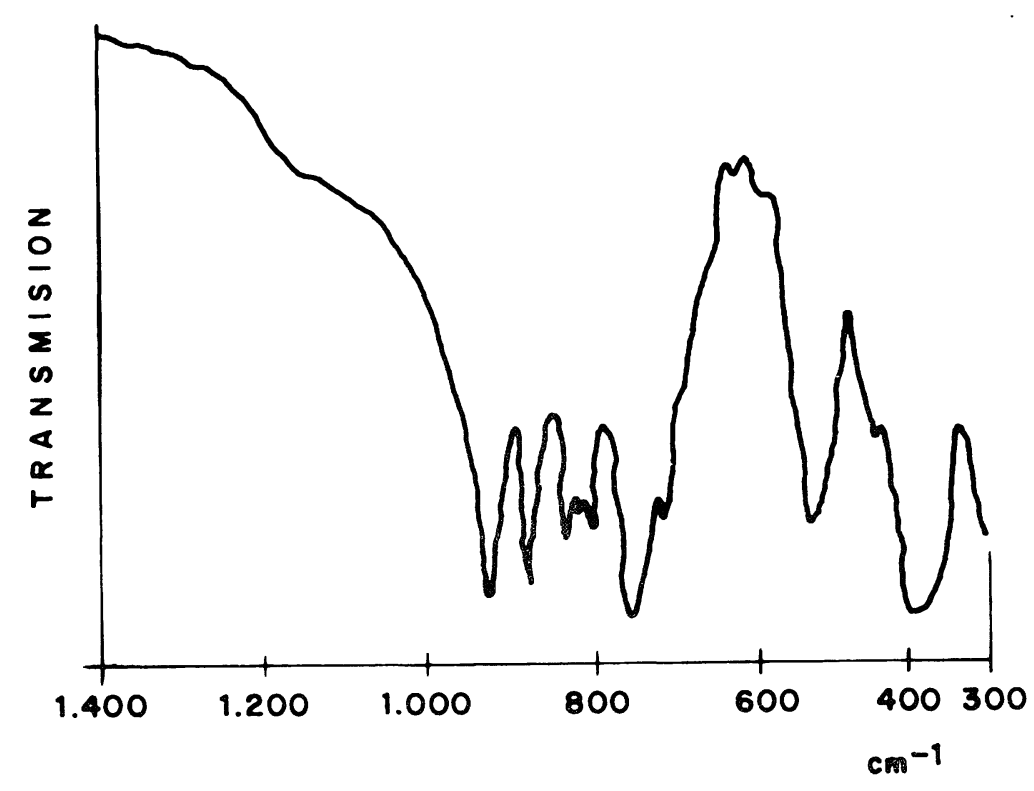

Fig. 6.-Espectro IR de $C_{y} A$.

CUADRO 2

Frecuencias de vibraciones en $\mathrm{cm}^{-1}$, de las bandas correspondientes al $C_{3} A$.

\begin{tabular}{cccc}
\hline FRECUENCIA $\left(\mathrm{cm}^{-1}\right)$ & ASIGNaCion & FReCUencia $\left(\mathrm{cm}^{-1}\right)$ & ASIGNACIOA \\
\hline $900 \mathrm{f}$ & & $620 \mathrm{~d}$ & $2 \times 310$ \\
$862 \mathrm{f}$ & $\nu_{3}$ & $588 \mathrm{~d}$ & no asignada \\
$842 \mathrm{~h}$ & & $536 \mathrm{~h}$ & \\
$817 \mathrm{~m}$ & $2 \nu_{2}(2 \times 412)$ & $534 \mathrm{~m}$ & $\nu_{4}$ \\
$800 \mathrm{~m}$ & $412+388$ & $512 \mathrm{~h}$ & no asignada \\
$786 \mathrm{~m}$ & $2 \nu_{2}(2 \times 388)$ & $464 \mathrm{~h}$ & no asignada \\
$758 \mathrm{~h}$ & no asignada & $456 \mathrm{~m}$ & \\
$740 \mathrm{~F}$ & $\nu_{1}$ & $412 \mathrm{f}$ & $\nu_{2}$ \\
$706 \mathrm{~m}$ & $388+310$ & $388 \mathrm{~F}$ & no asignada
\end{tabular}




\subsection{Estudio por IR del clínker portland}

La determinación cualitativa de las fases principales se realiza en base a las bandas de absorción ya descritas. Debe tenerse en cuenta que el espectro infrarrojo de una mezcla de sustancias da la superposición de los espectros individuales de cada uno de los compuestos. Por lo tanto, en el correspondiente al clínker portland, las fases componentes se solapan mutuamente. En todo caso las bandas de absorción específicas de cada componente, y por las que más fácilmente se detecta su presencia, son las siguientes (fig. 7):

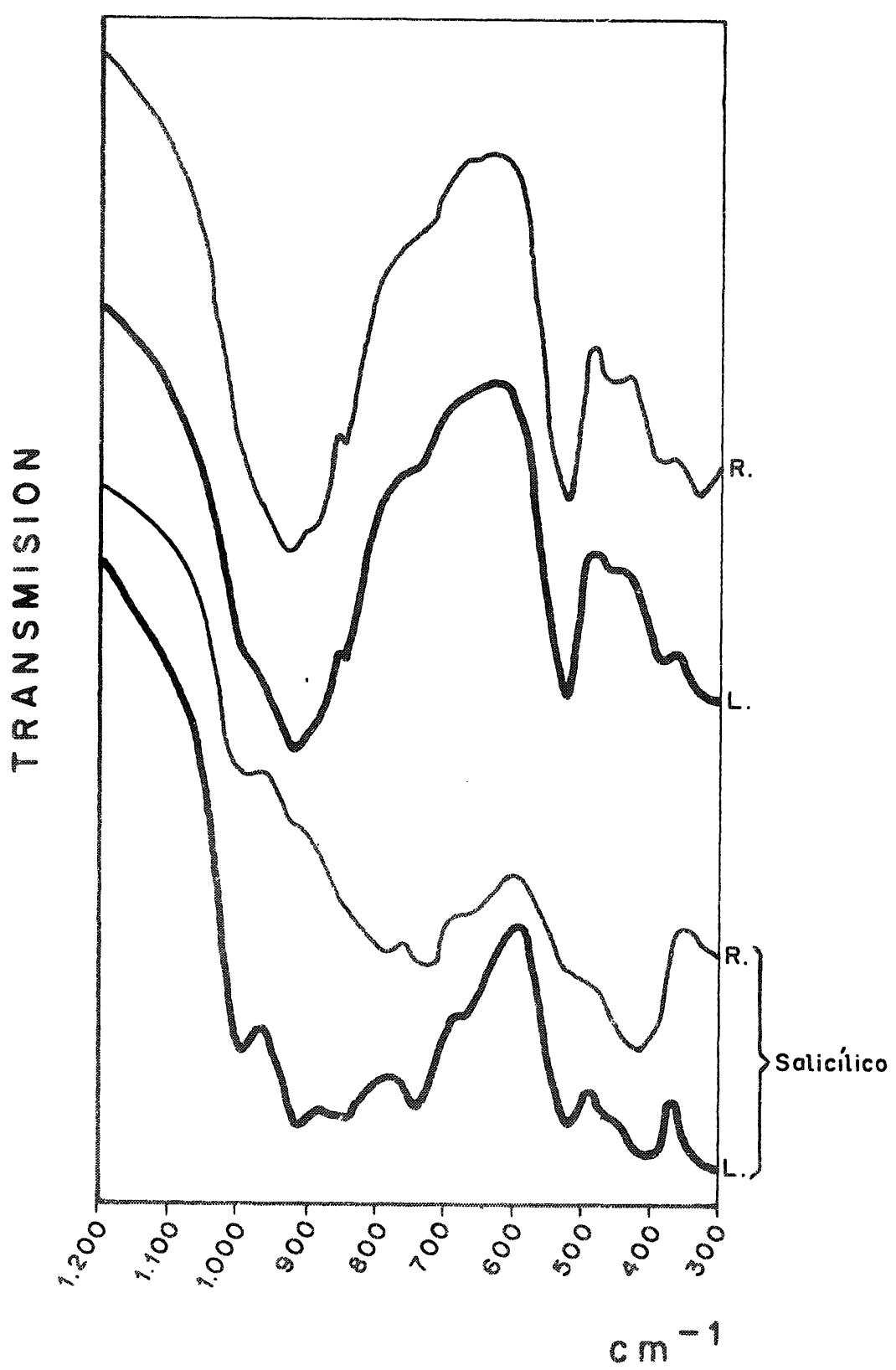

Fig. 7.-Espectros IR de clinker portland sintetizado en el laboratorio a partir de crudos Industriales.

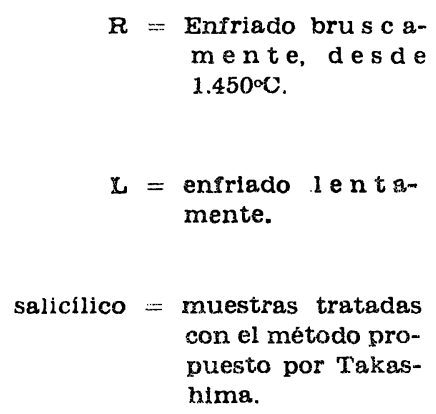

- Alita: banda muy intensa, ancha, hacia $920-930 \mathrm{~cm}^{-1}$.

- Beilia: banda débil (generalmente un "hombro") hacia $990 \mathrm{~cm}^{-1}$; se encuentra par. cialmente enmascarada por la de $920-930 \mathrm{~cm}^{-1}$.

- Fase Leritica: bandas muy débiles en la zona $600-700 \mathrm{~cm}^{-1}$. 
- Fase alumínica: bandas débiles en la zona $720-830 \mathrm{~cm}^{-1}$ (generalmente hacia $740 \mathrm{~cm}^{-1}$ ).

Como observaciones a tener en cuenta, están las siguientes: La existencia de sulfatos y/o carbonatos así como adiciones pueden dar lugar a confusión en la interpretación de las bandas específicas de las fases del clínker. Al no ser estas fases compuestos puros y definidos, los valores específicos dados anteriormente oscilan de una muestra a otra. La velocidad de enfriamiento de un clínker afecta (como era de suponer) a su espectro IR, ya que además de reflejar las distintas composiciones de las fases resultantes, dan unas bandas de absorción más o menos definidas, como consecuencia de una mayor o menor cristalinidad.

El estudio de las fases ferrítica y alumínica es mucho más efectivo si el clínker se somete previamente a un proceso de eliminación de silicatos. Para ello aconsejamos el de TAKASHIMA (19) que se realiza como sigue: se mezclan $0,5 \mathrm{~g}$ de clínker con $3 \mathrm{~g}$ de ácido salicílico y $20 \mathrm{ml}$ de metanol. Se agita (en agitador magnético) durante 30 minutos y se filtra a vacío.

En la figura 7 se dan los espectros correspondientes a, un clínker obtenido con enfriamiento brusco, con enfriamiento lento y sendos espectros de sus tratamientos por el método de TAKASHIMA.

P. TARTE (16) dice que la relación $\mathrm{Al} / \mathrm{Fe}$ de la fase ferrítica de un clínker podría estudiarse por espectroscopía IR de las dos formas siguientes:

a) Por la posición de las bandas, y

b) por la intensidad relativa de las mismas.

A continuación se resume el método propuesto:

a) Por la posición de las bandas

Mediante el estudio de la posición de dos bandas, una de ellas situada hacia $800-830 \mathrm{~cm}^{-1}$ y la otra hacia $700-730 \mathrm{~cm}^{-1}$, TARTE deduce que su posición varía entre 3 y $5 \mathrm{~cm}^{-1}$ para una variación de $x=0,05$ en la fórmula general de la fase ferrítica $C_{2}\left(A_{1-\mathrm{x}}, F_{\mathrm{x}}\right)$.

b) Por la intensidad relativa de las bandas

Las bandas de absorción en la zona de $600(A)$ y $700(B) \mathrm{cm}^{-1}$ varían en función del valor de $x$ de la fórmula $C_{2}\left(A_{1-\mathrm{x}}, F_{\mathrm{x}}\right)$, de tal modo que se pueden relacionar gráficamente. TARTE lo realiza representando en un eje de coordenadas el valor de $x$ frente a la relación:

$\frac{\log I_{0} / I(A)}{\log I_{0} / I(B)}$

siendo $I_{i}$, la intensidad de la radiación incidente e $I$ la intensidad de la radiación emitida $(*)$.

(*) Ver nota a pie de la página 28 del n." 175 de MATERIALES DE CONSTRUCCION. 
Indica el autor que las medidas de las intensidades de las bandas (método b) no es probable que conduzcan a conclusiones prácticas, entre otras causas por el solapamiento de bandas; por las diferentes velocidades de enfriamiento que pueden hacer variar la distribución del $\mathrm{Al}$ en posiciones tetraédricas u octaédricas; por la presencia de fases extrañas, etc.

En cuanto al método a) TARTE dice que es más correcto, al no influir en la posición de las bandas las mismas circunstancias descritas para el caso $b$ ).

Lógicamente lo idóneo para el análisis del clínker sería la determinación cuantitativa rápida de sus fases. Pensamos que este problema aún no está satisfactoriamente resuelto. No obstante describimos a continuación el método que proponen y desarrollan B. KASSNER y O. HENNING (19)

Para determinar cuantitativamente las cuatro fases principales del clínker (alita, belita, aluminatos, ferritos) se escogen, respectivamente, las seis bandas siguientes: [1] $650 \mathrm{~cm}^{-1}$; [2] $745 \mathrm{~cm}^{-1}$; [3] $999 \mathrm{~cm}^{-1}$; [4/1] $940 \mathrm{~cm}^{-1}$; [4/2] $450 \mathrm{~cm}^{-1}$; [4/3] $523 \mathrm{~cm}^{-1}$. A partir de la ley de LAMBER-BERT expresada como sigue:

siendo $E=$ extinción

$$
E=K_{\mathrm{v}} \cdot e
$$

$$
K_{\mathrm{v}}=\frac{K_{\mathrm{v}}^{\prime}}{F}
$$

$e=$ peso de la muestra.

$K_{\mathrm{v}}^{\prime}=$ coeficiente de extinción.

$F=$ superficie de los comprimidos (constante).

Se compone un sistema de cuatro ecuaciones con las extinciones resultantes de las medidas de las bandas [1], [2], [3] y [4]. Se deduce en porcentaje los contenidos de ferritos, aluminatos, belita y alita. Por cada análisis se hacen tres cálculos, ya que en la ecuación se va sustituyendo sucesivamente la extinción resultante de la banda [4/1] por la de la [4/2] y por la de la [4/3]. El resultado se da como promedio.

KASSNER Y HENNING dicen en su trabajo que de esta forma analizan 25 clínkeres de distinta procedencia. El tiempo exigido es del orden de 70 minutos por análisis.

Comparan los resultados con los obtenidos por microscopía óptica, difracción de rayos $\mathrm{X}$ y cálculo potencial. En el siguiente cuadro se dan los resultados que obtienen los autores por esas técnicas.

\begin{tabular}{lcrrc}
\hline Método de análisis (\% en peso) & IR & Micros. & Rayos $\mathbf{x}$ & Anal. Potencial \\
\hline Alita & 67 & 66 & 58 & 61 \\
Belita & 12 & 12 & 17 & 15 \\
Aluminatos & 14 & & 9 & 12,5 \\
Ferritos & 9 & 21 & 15 & 10 \\
\hline
\end{tabular}

Los resultados obtenidos son reproducibles. 
En el mismo trabajo KASSNER y HENNING dicen que una aproximación semicuantitativa puede hacerse sin cálculo alguno comparando los espectros con otros standard, o haciendo una estimación subjetiva cuando se posee suficiente experiencia.

Esta última observación la consideramos de gran utilidad y de fácil aplicación.

\subsection{Conclusiones prácticas}

- Por espectroscopía IR se puede realizar de forma inmediata el estudio cualitativo de las fases del clínker portland.

- Aun cuando la determinación cuantitativa es más compleja y problemática, se han propuesto métodos de trabajo mediante los cuales se obtienen valores fiables.

- Las determinaciones "semicuantitativas" (valores relativos) son rápidas y eficaces.

- La forma del perfil de las bandas IR da una indicación respecto a la velocidad de enfriamiento de la muestra correspondiente.

- La espectroscopía IR es una técnica indicada para el estudio de las fases del clínker (de manera especial ferritos y aluminatos) que pueden formarse con pobre cristalinidad.

(Continuará) 